\title{
A doença arterial coronariana e o envelhecimento populacional: como enfrentar esse desafio?
}

\author{
Coronary artery disease and populational ageing: \\ how to deal with this challenge?
}

\section{Resumo}

O envelhecimento populacional é um desafio do mundo contemporâneo que afeta tanto os países ricos quanto os pobres. Além da sua alta prevalência, a doença arterial coronariana em populações idosas está associada à maior frequência de apresentações clínicas atípicas, a peculiaridades na investigação diagnóstica e à necessidade de cuidado redobrado na abordagem terapêutica. No Brasil, as doenças do aparelho cardiovascular constituem as principais causas de mortalidade, seguidas por neoplasias, causas externas e doenças do aparelho respiratório. No idoso, as manifestações clínicas da doença arterial coronariana podem ser diferentes e atípicas, sendo a dispneia de esforço mais comum que a angina pectoris. A investigação complementar é semelhante a da população geral, mas a acurácia dos diversos métodos pode variar bastante. As diferenças na fisiopatologia, farmacocinética e metabolismo das drogas observadas no idoso devem ser consideradas ao se estabelecer uma estratégia de tratamento e os alvos terapêuticos.
Para o controle dos múltiplos fatores de risco e comorbidades, é necessário o uso de maior número de fármacos, ocasionando interações medicamentosas, reações adversas, menor adesão, maior custo e hospitalizações por complicações iatrogênicas. Para minimizar os efeitos colaterais decorrentes da associação de múltiplos medicamentos, assim como aumentar a adesão, deve-se revisar as prescrições a cada consulta, ressaltar a importância de cada uma das substâncias prescritas, ajustar as doses e a forma de administração, procurando-se reduzir a sua frequência. Efeitos colaterais podem agravar ou sugerir a presença de outros distúrbios clínicos. As decisões clínicas envolvendo o tratamento da doença arterial coronariana devem considerar a fragilidade, a expectativa e a qualidade de vida, assim como os riscos e os custos. As estratégias terapêuticas e diagnósticas farmacológicas ou invasivas devem ser criticamente selecionadas face ao maior potencial iatrogênico observado nos pacientes idosos.

Descritores: Doença das coronárias; Idoso; Diagnóstico; Terapêutica. 


\section{Abstract}

Ageing is a challenge in contemporary world affecting rich and poor countries. Besides its high prevalence, coronary artery disease in elderly is associated to more atypical presentations, singularities in clinical investigation and a more careful therapeutic approach.

In Brazil, cardiovascular diseases are the major causes of death, followed by cancer, external causes and respiratory diseases. The clinical presentations of coronary artery disease may be different and atypical and dyspnea is more common than angina pectoris. Subsequent investigation is similar to younger people but the accuracy of many methods may vary a lot.

The differences in the pathophysiology, pharmacokinetics and metabolism of drugs observed in older people must be considered when selecting a therapeutic strategy and its targets. More drugs are necessary to control multiple risk factors and comorbidities, leading to more drug interactions, side effects, less adherence to therapy, higher costs and hospitalizations due to iatrogenic complications. In a way to minimize the side effects due to the association of multiple drugs, prescriptions must be revised in each interview, emphasizing the importance of each drug, adjusting doses and administration routes and aiming to reduce the frequency of administrations. Side effects may exacerbate or suggest other diseases. Clinical decision related to the treatment of coronary artery disease must consider frailty, quality of life and life expectancy, as well as risk and costs. Pharmacological and invasive strategies must be critically selected due to their higher iatrogenic potential observed in the elderly.

Keywords: Coronary disease; Aged; Diagnosis; Therapeutics.

\section{Introdução}

O envelhecimento é um desafio do mundo atual que afeta tanto os países ricos quanto os pobres. Além da sua alta prevalência, a doença arterial coronariana (DAC) em populações idosas está associada à maior frequência de apresentações clínicas atípicas, a peculiaridades na investigação diagnóstica e à necessidade de cuidado redobrado na abordagem terapêutica.

\section{Epidemiologia}

O processo de envelhecimento da população mundial tem as suas origens enraizadas nas transformações socioeconômicas vividas pelas nações desenvolvidas no século passado, e que, no entanto, só produziram modificações significativas nas suas variáveis demográficas na virada no século $20{ }^{1,2}$ Seguindo os padrões observados em outros países, no Brasil a queda da fecundidade e o aumento da expectativa de vida resultaram nos últimos 40 anos no aumento absoluto e relativo da população idosa.

As doenças não transmissíveis são atualmente as principais responsáveis pela carga de doenças que acometem a população mundial, um fenômeno associado às transformações demográfica e epidemiológica que acompanharam a crescente urbanização, o desenvolvimento tecnológico e a redução das taxas de fertilidade e de mortalidade. As doenças cardiovasculares constituem hoje quase metade das doenças não transmissíveis, sendo responsáveis por 17,3 milhões de mortes por ano, um número que em torno de 2030 deverá aumentar para mais de 23,6 milhões. De maneira crescente, as populações afetadas são aquelas que vivem em países de renda baixa ou média, onde ocorrem $80 \%$ dessas mortes, comumente em indivíduos mais jovens do que aqueles acometidos nos países de alta renda. ${ }^{3}$

As perdas econômicas cumulativas por doenças não transmissíveis nos países de renda baixa e média projetadas para o período 2011 a 2025 serão de US\$ 7,28 trilhões, e estima-se que o custo global de não se investir em prevenção e tratamento de doenças cardiovasculares será de US\$ 47 trilhões, nos próximos 25 anos. ${ }^{3}$

Em 2009 no Brasil as doenças do aparelho circulatório foram as principais causas de óbitos (29\%), seguidas por neoplasias, causas externas e doenças do aparelho respiratório, que juntas 
totalizaram $60 \%$ das mortes naquele ano. ${ }^{4}$

A faixa etária de 40 a 59 anos apresenta uma transição em relação às anteriores. No sexo masculino, a principal causa de óbito foram as doenças do aparelho circulatório, com $25 \%$ das mortes; as causas externas apareceram em segundo lugar, com $18 \%$; e as neoplasias com $16 \%$. No sexo feminino as principais causas de óbito foram as neoplasias (29\%) e as doenças do aparelho circulatório (28\%). Finalmente, os óbitos de 60 anos e mais, em ambos os sexos, ocorreram pelas mesmas causas e na mesma ordem, com percentuais semelhantes: doenças do aparelho circulatório (37\%), neoplasias (17\%) e doenças do aparelho respiratório (13\%). ${ }^{4}$

A DAC é a causa mais comum de morte na maior parte dos países afluentes da Europa e América do Norte, ocupando a segunda posição no Brasil, onde é suplantada apenas pelas doenças cerebrovasculares. ${ }^{4,5}$ Nos Estados Unidos, mais de $80 \%$ das mortes por DAC ocorre em pessoas com mais de 65 anos de idade, assim como $37 \%$ dos infartos agudos do miocárdio diagnosticados ocorrem em indivíduos com 75 anos ou mais. Do ponto de vista estatístico, $40 \%$ das DACs têm sua primeira manifestação como infarto agudo do miocárdio (IAM) e 10\% a 20\% dos casos se apresentam como morte súbita. Considera-se que os quadros clínicos sintomáticos de DAC são a ponta do iceberg de um grande número de idosos com formas assintomáticas e subclínicas da condição. ${ }^{6}$

\section{Quadro clínico: peculiaridade e equivalentes anginosos}

O espectro da DAC inclui formas assintomáticas ou subclínicas, angina pectoris estável crônica, angina instável e infarto agudo do miocárdio. ${ }^{6}$

A DAC é habitualmente diagnosticada em pessoas idosas diante de evidências de DAC significativa em uma angiografia coronariana, um infarto do miocárdio documentado, uma história típica de angina pectoris com isquemia miocárdica diagnosticada por teste de estresse, ou por morte cardíaca súbita. ${ }^{5}$
Quanto às manifestações clínicas em indivíduos idosos, a dispneia é muito mais comum do que a dor torácica típica de angina pectoris. Nestes casos, a dispneia costuma ser de esforço e está relacionada a um aumento transitório da pressão diastólica final do ventrículo esquerdo, causada por isquemia sobreposta a uma complacência reduzida do ventrículo esquerdo. ${ }^{5}$ Ainda, como idosos costumam ter limitações funcionais e de atividades físicas mais frequentemente do que indivíduos mais jovens, a angina pectoris é menos associada a exercícios; eles se queixam menos de dor subesternal, e costumam descrever a dor como sendo de menor intensidade e de duração mais curta. Pode também ocorrer como queimação epigástrica pós-prandial, dor torácica posterior ou nos ombros. Edema pulmonar agudo pode ser uma manifestação de angina instável produzida por extensa DAC em idosos. ${ }^{5}$ Naturalmente, estas apresentações atípicas da DAC obrigam-nos ao diagnóstico diferencial com outras condições mórbidas, frequentes em indivíduos idosos, tais como doença degenerativa das articulações, úlcera péptica e hérnia de esôfago.

A isquemia miocárdica silenciosa ou assintomática é outra apresentação frequentemente observada em idosos.

\section{Métodos complementares: há diferença na acurácia?}

Grande parte dos idosos portadores de DAC evolui de forma assintomática ou com equivalentes anginosos, o que pode atrasar o início do tratamento e aumentar a mortalidade cardiovascular. Dessa forma, a solicitação de exames complementares, para o diagnóstico e estratificação de risco, se torna fundamental para essa classe de pacientes, propiciando uma detecção mais precoce da doença., ${ }^{7,8}$

\section{Eletrocardiograma (ECG)}

As recomendações são semelhantes para qualquer paciente com suspeita de DAC. $\mathrm{Na}$ presença de quadro sugestivo de angina estável, todo paciente deve realizar um ECG de repouso, 
embora aproximadamente $50 \%$ dos casos tenha resultado normal, o que pode ocorrer mesmo em pacientes que possuam DAC grave. ${ }^{8,9}$

\section{Teste ergométrico (TE)}

Há uma preocupação frequente em relação a eventuais riscos de lesões articulares desencadeados pela atividade durante o TE, além de outros problemas como instabilidade postural, incapacidade cognitiva, pouca familiaridade com a esteira, medo, ansiedade e falta de motivação. Esse conjunto de fatores pode dificultar a realização do TE no idoso, motivando o clínico, muitas vezes, a preferir a realização de exames de imagem com estresse farmacológico, como a cintilografia, em detrimento de TE. ${ }^{7}$ Não há, contudo, uma clara razão para contraindicar o teste ergométrico em idosos, ${ }^{10,11}$ já que se trata de exame com boa acurácia para o diagnóstico de DAC. As diretrizes norte-americanas estimam que, para diagnóstico de DAC em idosos, o TE tenha maior sensibilidade (84\%) e menor especificidade (70\%) quando os seus resultados são comparados com aqueles observados em indivíduos jovens. ${ }^{10}$

A avaliação de idosos pelo TE segue os critérios estabelecidos para os exames em geral, e a indicação mais recomendada é para o diagnóstico de isquemia miocárdica, além da avaliação da capacidade funcional. Os protocolos de baixa carga são os mais utilizados para pacientes mais idosos. ${ }^{9}$

O TE é, portanto, seguro, factível, custo-eficaz e até mesmo superior à cintilografia miocárdica com dipiridamol na predição do risco de DAC em pacientes idosos. ${ }^{7}$ Além disso, em nosso contexto social, devido a sua boa relação risco-benefício, salvo suas tradicionais limitações, o TE é apropriado como primeiro exame complementar a ser solicitado na pesquisa de isquemia miocárdica. ${ }^{12}$

\section{Cintilografia miocárdica $(\mathrm{CM})$}

A CM é um exame seguro e não invasivo, com alta acurácia para o diagnóstico de DAC, através da avaliação direta da perfusão miocár- dica, ${ }^{13}$ apresenta evidências que demonstram resultado igualmente eficaz nos idosos, quando comparado à sua aplicação em populações mais jovens. ${ }^{14,15}$ Entretanto, em função das limitações já citadas, a proporção de cintilografias miocárdicas solicitadas, em comparação com o TE, cresce com a idade dos indivíduos avaliados. ${ }^{16} \mathrm{~A}$ adição das imagens cintilográficas perfusionais ao TE aumenta a sensibilidade para detecção de DAC para $90 \%$, com uma especificidade de $87 \%{ }^{13}$

\section{Ecocardiograma (ECO)}

O ECO de repouso é um exame seguro, de baixo custo e altamente disponível, devendo ser realizado em indivíduos com suspeita de DAC e também para estratificação de risco em pacientes com a doença conhecida, além de ser capaz de sugerir outras causas de dor precordial, como dissecção aórtica, pericardite, embolia pulmonar ou doenças orovalvares, como a estenose aórtica. ${ }^{17} \mathrm{O}$ exame é capaz de identificar, ainda, marcadores de risco subclínicos, como avaliação da função ventricular, aumento atrial ou hipertrofia ventricular, que agregam informacões importantes na estratificacão de risco dos pacientes idosos. ${ }^{18,19}$

O ECO com estresse, sob esforço físico ou sob ação de drogas vasodilatadoras (dipiridamol) ou estimulantes adrenérgicas (dobutamina), também possui boa acurácia para a detecção de isquemia miocárdica induzida em pacientes com probabilidade pré-teste intermediária ou alta. ${ }^{17,19}$ Devido a limitações de esforço físico, frequentes nesse grupo de pacientes, o estresse farmacológico se torna opção de escolha na maioria das vezes. ${ }^{9,20}$ A frequência de complicações durante o exame é bastante baixa, sendo menor nos exames com exercício e raros casos cursam com IAM ou óbito. ${ }^{9}$

Em diversos estudos que avaliam a acurácia do exame foram encontrados valores médios de sensibilidade de $88 \%$ e especificidade de $83 \%$ para a presença de estenose coronária acima de $50 \%{ }^{21}$ 
Ressonância magnética cardíaca (RMC)

Apresenta grande utilidade na avaliação de forma completa da doença arterial coronária, tanto em sua fase aguda como crônica. O método é capaz de avaliar a função global e regional, detectar e quantificar áreas de IAM. Isto permite à RMC grande acurácia na determinação da viabilidade miocárdica, além de possibilitar uma completa avaliação da isquemia através de técnicas de perfusão em estresse, trazendo ao mesmo tempo informações diagnósticas e prognósticas. ${ }^{9,22}$

Um trabalho recente demonstrou níveis de sensibilidade e especificidade em torno de $83 \%$ e $86 \%$, respectivamente. ${ }^{23}$ Os protocolos utilizados empregam estímulo farmacológico com dobutamina ou, em geral, dipiridamol para induzir a isquemia miocárdica. Esta última é contraindicada em portadores de doença pulmonar obstrutiva, uma condição mais comum nos idosos do que nos pacientes mais jovens, $o$ que faz com que centros especializados deem preferência à utilização de dobutamina neste subgrupo. $^{22,23}$

É importante lembrar que os compostos de gadolínio, base dos contrastes da ressonância magnética, são atualmente contraindicados nos pacientes com redução importante da função renal - que é mais frequente em pacientes idosos - porque nestes pode haver o desenvolvimento de fibrose nefrogênica sistêmica, doença rara, mas com evolução grave e habitualmente fatal, sem tratamento estabelecido.

Angiotomografia de artérias coronárias

É um exame que permite a avaliação anatômica, útil para excluir a presença de doença coronariana; portanto, possui alto valor preditivo negativo. Possui maior valor diagnóstico em pacientes com probabilidade intermediária de DAC e com testes funcionais não diagnósticos ou conflitantes. Também tem maior valor em pacientes com baixa probabilidade pré-teste de DAC mas com teste funcional positivo. ${ }^{9,22}$
O escore de cálcio é utilizado para estratificação de risco, representando a extensão de doença coronária em um determinado indivíduo. Deve ser utilizado em pacientes oligossintomáticos ou assintomáticos, com risco intermediário de eventos cardiovasculares pelos fatores de risco tradicionais, ou com histórico familiar de doença coronária precoce na família. ${ }^{22}$

Em pacientes muito idosos e/ou com DAC avançada, a indicação da angiotomografia é mais limitada, ${ }^{24}$ já que a calcificação coronária que acompanha o processo de envelhecimento dificulta a visualização da luz vascular, diminuindo a sensibilidade e a especificidade do método. ${ }^{9}$ Entretanto, o exame tem sua utilidade quando os exames não invasivos têm resultados discordantes, ou para pacientes com dificuldades de realizar esforço físico e que possuem comorbidades que limitam a realização de outros exames indutores de isquemia. ${ }^{9}$ Importante lembrar que a presença de disfunção renal também é fator limitante à realização do exame, já que é feito com a utilização de contraste iodado.

\section{Coronariografia}

É um exame capaz de revelar toda a anatomia coronariana do paciente, demonstrando a presença de lesões obstrutivas e de quantificá-las, auxiliando a decisão terapêutica.

Apesar do maior risco de complicações, não há diferenças em termos de resultados na realização da coronariografia entre pacientes idosos ou mais jovens. O exame é mais comumente realizado no contexto da doença arterial coronariana crônica, na presença de exame não invasivo demonstrando alto risco para DAC, ou ainda para pacientes com DAC conhecida que não responderam ao tratamento conservador, com angina CCS III ou IV. Outras indicações são: a investigação de insuficiência cardíaca em determinados casos; em pacientes com angina estável que tiveram morte súbita abortada; e ainda, pacientes em pré-operatório de cirurgia de troca valvar. ${ }^{9}$

Por se tratar de um exame invasivo associado a riscos, é importante saber identificar que 
tipo de paciente realmente se beneficiará da sua realização. Especialmente em pacientes com mais de 60 anos, o risco de óbito relacionado ao exame é duas vezes superior à população geral, independente da presença de outras comorbidades. ${ }^{9}$

Uma complicação importante relacionada à realização desse exame em idosos é a nefropatia por contraste. A idade avançada é fator de risco independente para ocorrência dessa complicação. ${ }^{9}$ Outra complicação é o maior risco de sangramento durante e após o exame, que também é mais frequente nesse grupo de pacientes. Alguns estudos demonstraram menor risco de sangramento utilizando a via radial para realização do exame.

\section{Tratamento farmacológico e estratégias de revascularização no idoso}

O aumento na expectativa de vida associado a mudanças comportamentais tem colocado a população e a classe médica frente a um dilema: tendo em vista o objetivo de melhorar a qualidade de vida e produzir um impacto positivo no controle da morbimortalidade por DAC, qual é a melhor abordagem terapêutica para o indivíduo idoso?

Os idosos constituem uma população com peculiaridades fisiopatológicas, farmacocinéticas e biometabólicas que devem ser consideradas ao se estabelecer uma estratégia de tratamento e alvos terapêuticos. Para o controle dos múltiplos fatores de risco e comorbidades, é necessário o uso de maior número de fármacos (polifarmácia) ocasionando interações medicamentosas, reações adversas, menor adesão, maior custo e hospitalizações por complicações iatrogênicas. Além disso, ao prescrever para a população idosa, deve-se considerar as limitações cognitivas, a menor acuidade visual, o nível de independência e a relação com a família. Devemos estar atentos aos efeitos do uso concomitante de múltiplos fármacos, que muitas vezes geram efeitos colaterais que são interpretados como um novo sinal ou sintoma clínico e acabam recebendo tratamento adicional.

Visando minimizar os efeitos colaterais da associação de múltiplos medicamentos, assim como aperfeiçoar a adesão, cabe ao médico a preocupação de revisar as medicações a cada consulta, ressaltar a importância de cada uma das substâncias prescritas, verificar se as doses prescritas estão corretas, assim como o modo de administração, e preferencialmente reduzir a frequência das tomadas. Ao médico cabe, ademais, evitar prescrições que possam agravar outras enfermidades, manter uma única receita com todos os medicamentos e solicitar a presença dos familiares, quando necessário. A melhor adesão proporciona menor risco de hospitalização por qualquer causa. ${ }^{25}$

A decisão clínica envolvendo o tratamento da DAC deve ponderar os custos, os benefícios, a qualidade de vida e os riscos, buscando estabelecer a melhor estratégia terapêutica - farmacológica ou invasiva - proporcionando aumento da sobrevida, redução dos sintomas e aumento da expectativa de vida.

\section{Drogas antiplaquetárias}

A aspirina na dose de 75 a $325 \mathrm{mg} /$ dia reduz a mortalidade em $33 \%$ dos pacientes com DAC. A maioria dos estudos contempla o uso de aspirina na síndrome coronariana aguda (SCA), na qual é preconizado ataque de $162 \mathrm{mg}$ a 325 $\mathrm{mg}$, produzindo rápido efeito antitrombótico por inibir a produção de tromboxano A2, com significativa redução da mortalidade. Após o evento agudo, a manutenção se dá com 75 a 150 $\mathrm{mg} /$ dia; especialmente no idoso, doses maiores aumentam o risco de efeitos colaterais gastrointestinais e sangramentos.

A ticlopidina e o clopidogrel podem substituir a aspirina nos pacientes com hipersensibilidade ou intolerância à aspirina. A ticlopidina pode induzir neutropenia e, menos frequentemente, púrpura trombocitopênica trombótica, tendo um maior potencial de toxicidade e devendo ser evitada. O clopidogrel teve seus benefícios na redução de eventos cardiovasculares na 
SCA sem supra de ST (SCAsST) demonstrados no estudo Clopidogrel in Unstable Angina to Prevent Recurrent Events (CURE), no qual a associação com aspirina foi benéfica em reduzir a incidência de infarto agudo do miocárdio (IAM), acidente vascular cerebral (AVC) e morte cardíaca, quando comparada com o grupo que usou aspirina associado ao placebo. ${ }^{26}$ Nesse estudo utilizou-se dose de ataque de $300 \mathrm{mg}$, seguido por $75 \mathrm{mg}$, diariamente. Em pacientes submetidos à angioplastia, estudos relataram evolução favorável com dose de ataque de 600 mg comparado com $300 \mathrm{mg}$ (ARMYDA 2). ${ }^{9}$ Nos idosos, entretanto, um ataque com dose dobrada de $600 \mathrm{mg}$ ocasionou um maior risco de sangramento, sendo prudente manter a dose de ataque de $300 \mathrm{mg}$ para pacientes com 75 anos ou mais submetidos à angioplastia. Tal dose deverá ser mantida por 1 ano.

Vale ressaltar que muitos fitoterápicos de uso comum entre a população idosa exercem efeitos sobre a metabolização e efeito da aspirina. A castanha da índia interage tanto com a ticlopidina quanto com a aspirina, potencializando seus efeitos. Com interação semelhante também podemos citar ginkgo biloba e valeriana. ${ }^{27}$

\section{Nitratos}

Os nitratos não são fármacos com impacto na mortalidade, contudo diminuem a intensidade e a frequência dos episódios dolorosos, promovendo melhor qualidade de vida. Em geral, os idosos toleram bem os nitratos, embora a hipotensão e a cefaleia possam tornar difícil o seu uso em alguns pacientes, bem como o desenvolvimento de tolerância à droga. A hipotensão pode ser amenizada pela redução da dose, correção da hipovolemia e cuidados posturais ao se levantar. Para prevenir a tolerância à droga é recomendado um intervalo de 12 a 14 horas sem a sua utilização.

Os nitratos estão contraindicados em pacientes com pressão arterial sistólica menor que $90 \mathrm{mmHg}$, bradicardia, taquicardia e no infarto de ventrículo direito (VD).
Deve-se sempre avaliar se o paciente faz uso de inibidores da fosfodiesterase- 5 empregados no tratamento da disfunção erétil, em virtude do potente efeito hipotensor dessa associação.

\section{Betabloqueadores}

São recomendados como drogas de primeira escolha na DAC, particularmente em pacientes com IAM prévio, pelo considerável impacto na mortalidade. $\mathrm{O}$ tratamento deve ser iniciado com baixas doses.

Nos idosos é importante observar os efeitos colaterais: letargia, insônia, piora da claudicação, má tolerância ao exercício, fadiga, impotência, depressão, alterações de humor e alterações do sono. A bradicardia e o broncoespasmo são os mais temidos. Na presença de bradicardia ou disfunção do nó sinoatrial são contraindicados (exceto se implantado marcapasso). Devem ser usados com cautela em diabéticos, portadores de doença vascular periférica grave, depressão e em pacientes com asma ou DPOC, embora essas contraindicações não sejam absolutas e possam ser atenuadas pelo uso de betabloqueadores cardiosseletivos. Os betabloqueadores não devem ser iniciados em pacientes com insuficiência cardíaca descompensada. ${ }^{28}$

\section{Bloqueadores dos canais de cálcio (BCC)}

Não há evidências que comprovem a redução da mortalidade e de IAM em idosos com a utilização de bloqueadores dos canais de cálcio (BCC). Portanto, não devem ser utilizados como primeira escolha. Podem ser usados como terapia inicial, quando houver contraindicação aos betabloqueadores ou em associação para controle clínico da dor anginosa recorrente e hipertensão coexistente.

Os di-hidropiridínicos, como a anlodipina e a nifedipina, apresentam menor efeito inotrópico negativo e não inibem o nó sinoatrial e a condução atrioventricular, podendo ser usados em pacientes com disfunção sistólica ou em associação com betabloqueadores, quando estes forem ineficazes como monoterapia. Apresen- 
tam efeito vasodilatador coronariano e periférico importante, principalmente os de primeira geração, como a nifedipina, o que pode agravar os sintomas isquêmicos devido ao fenômeno do "roubo coronariano".

O verapamil e o diltiazem são potentes inibidores do nódulo sinoatrial e da condução atrioventricular, além de serem vasodilatadores periféricos e inotrópicos negativos. São contraindicados na vigência de IC sistólica, disfunção do nódulo sinoatrial e bloqueios atrioventriculares, devendo ser usados com extrema cautela quando combinados com betabloqueadores. O edema periférico associado com os BCCs é um efeito adverso relativamente comum, sobretudo nos idosos, e está relacionado com a redistribuição de fluido no espaço intersticial.

\section{Inibidores da enzima conversora da angiotensina}

Estudos clínicos demonstram que os inibidores da enzima conversora da angiotensina (IECAs) são benéficos no tratamento da doença coronariana, não só reduzindo a mortalidade, mas também prevenindo eventos coronarianos futuros, incluindo novo IAM. ${ }^{29}$

O estudo Heart Outcomes Prevention Evaluation (HOPE) ${ }^{29}$ avaliou o efeito do ramipril em idosos acima de 70 anos, observando a redução de eventos cardiovasculares maiores, IAM, AVC e mortalidade por todas as causas. Os resultados em pacientes com idade maior que 65 anos foram melhores do que nos mais jovens. Portanto, o uso de IECA deve ser considerado no tratamento de idosos com isquemia miocárdica, independente de sintomas de disfunção ventricular. $^{29}$

Os efeitos colaterais mais consideráveis no uso dos IECAs são hipotensão, tontura, anorexia, hipercalemia e tosse irritativa. Este último é o efeito adverso mais comum, aliviado com a interrupção do medicamento e substituição por bloqueadores do receptor da angiotensina (BRAs).

Pode haver aumento da creatinina sérica, efeito favorecido pela elevada prevalência de disfunção renal na população idosa. Nos casos de aumentos séricos da creatinina ( $>3 \mathrm{mg}$ / $\mathrm{dl})$, hipercaliemia (> 5,5 $\mathrm{mEq} / \mathrm{l})$ e suspeita de estenose das artérias renais bilaterais, o uso de IECAs é contra indicado. Os BRAs constituem uma alternativa terapêutica para pacientes que não podem ser tratados com IECAs. ${ }^{28}$

\section{Estatinas}

Estudos comprovam a eficácia do uso de estatina na prevenção primária e secundária em idosos. O estudo Heart Protection Study (HPS) demonstrou uma redução na mortalidade e na incidência de primeiro infarto e de AVE, em todas as faixas etárias, incluindo octogenários em uso de estatina, tanto para a prevenção primária quanto secundária. ${ }^{30}$

O Prospective Study of Pravastatin in the Elderly at Risk (PROSPER), que utilizou a pravastatina na dose de $40 \mathrm{mg}$ ao dia, foi o único desenhado para avaliar o benefício da redução do colesterol e suas frações, especificamente na população idosa. Houve uma redução de 19\% no risco relativo envolvendo morte ou IAM não fatal nos pacientes tratados. Não demonstrou benefício na redução do risco de AVE. Idosos com baixo HDL-c apresentaram maiores benefícios. $^{31}$

No idoso sem DAC estabelecida, mas com alto risco para doença coronariana, deve-se realizar prevenção primária com estatina, como recomendado no Programa Nacional de Educação em Colesterol III (NCEP III). ${ }^{9}$

Em pacientes admitidos por SCA, o estudo MIRACL ${ }^{32}$ demonstrou que a redução precoce e agressiva do LDL-c com atorvastatina na dose de $80 \mathrm{mg}$ por dia reduziu a incidência de eventos isquêmicos recorrentes nas primeiras 16 semanas de SCAsSST. Esse estudo selecionou 3.086 pacientes com idade média de 65 anos. ${ }^{28,32}$

\section{Cirurgia de revascularização do miocárdio e intervenção coronariana percutânea no idoso}

A idade é um fator que deve ser considerado 
na indicação de cirurgia de revascularização do miocárdio (CRVM). A morbidade e a mortalidade aumentam progressivamente com a idade, tanto na CRVM como na intervenção coronariana percutânea (ICP). Idosos apresentam peculiaridades e um número maior de comorbidades que se somam para aumentar o risco operatório dessa população.

Estudos mostraram que a CRVM no idoso traz consequências adversas, que ocasionam um aumento na mortalidade intra-hospitalar, no desenvolvimento de insuficiência renal e de AVE, quando comparadas aos mais jovens. A presença de ateroma na aorta ascendente e arco aórtico, assim como obstruções significativas em carótidas, constituem um importante fator de risco para AVE nos pacientes que são submetidos à circulação extracorpórea (CEC) e consequente canulação da aorta. ${ }^{28,33} \mathrm{~A}$ incidência é de aproximadamente $6 \%$ para lesões neurológicas graves e $57 \%$ para déficit cognitivo leve, ocasionados tanto por hipoperfusão durante a CEC, quanto por fenômenos embólicos. ${ }^{32}$ A diretriz ACC/ AHA refere mortalidade de 5,28\% para pacientes acima de 75 anos e $8,38 \%$ em octagenários. ${ }^{15}$

Com os avanços técnicos, envolvendo procedimentos minimamente invasivos e a possibilidade de cirurgia sem CEC, houve uma diminuição do risco associado à cirurgia em indivíduos idosos. Isto porque foram minimizados os efeitos inflamatórios e as complicações pós-operatórias como AVE, delirium, distúrbios cognitivos e fibrilação atrial. Entretanto, além de ser uma cirurgia mais trabalhosa, pode haver comprometimento dos resultados, por uma menor patência dos enxertos e revascularização incompleta.

A Sociedade Internacional de Cirurgia Cardiotorácica Minimamente Invasiva recomenda que a CRVM sem CEC deva ser considerada para reduzir a mortalidade e a morbidade em pacientes de alto risco (idade $>75$ anos, diabetes, insuficiência renal, disfunção ventricular esquerda e Euroscore $>5$ ). ${ }^{34}$

Em virtude da descalcificação característica do envelhecimento, a esternotomia mediana completa pode aumentar os riscos pós-operatórios de deiscência do esterno e infecções, aumentando o tempo de ventilação mecânica e de internação.

Embora haja evidências de que a CRVM apresenta vantagens em relação à ICP nos pacientes multiarteriais, deve-se considerar que no idoso a CRVM associa-se a maior mortalidade hospitalar. Assim, na população geriátrica devemos individualizar a melhor estratégia de reperfusão, considerando a presença de comorbidades, gravidade da doença, possibilidade ou não de ICP, expectativa de vida e a vontade do paciente.

A indicação da ICP no idoso aumentou nos últimos anos com os avanços tecnológicos e o surgimento dos stents farmacológicos, sendo atualmente uma opção para lesões complexas e para o tratamento de doenças multiarteriais e SCA em qualquer faixa etária. Pacientes maiores de 70 anos, excluídos das indicações iniciais de ICP, atualmente são abordados por meio desse método.

O uso de stents farmacológicos, mais eficazes na prevenção da reestenose e nova revascularização, permitiu a ampliação do espectro de indicação para os casos predispostos às recidivas, como os diabéticos e os que apresentam lesões em vasos de fino calibre e lesões reestenóticas. Contudo, o uso de stent farmacológico implica em dupla antiagregação plaquetária com aspirina e clopidogrel por no mínimo 1 ano. Essa determinação impõe maiores riscos ao paciente idoso, não apenas por aumentarem o risco de sangramento e complicações hemorrágicas, mas também pelo fato de apresentarem comorbidades associadas que exigem a descontinuação da terapêutica. ${ }^{35}$

\section{Estratégia invasiva ou conservadora pós-SCA}

O estudo Trial of Invasive versus Medical therapy in Elderly patients (TIME) ${ }^{36}$ foi o primeiro estudo prospectivo randomizado que comparou tratamento medicamentoso otimizado com a estratégia invasiva (CRVM e ICP) 
em pacientes com idade superior a 75 anos e angina estável. No estudo, $72 \%$ dos pacientes foram tratados por meio de ICP e apresentaram reduções significativas de angina, número de medicamentos antianginosos utilizados, re-hospitalizações e necessidade de procedimentos adicionais de revascularização, após 6 meses de seguimento. Nos 4 anos seguintes, a sobrevida foi similar em ambas as estratégias e a ausência de revascularização no primeiro ano foi um preditor independente de maior mortalidade. Os pacientes da estratégia invasiva também apresentaram sobrevida livre de eventos cardíacos significativamente menores. ${ }^{37}$

O estudo Clinical Outcomes Utilizing Revascularization and Agressive Drug Evaluation (COURAGE), ${ }^{38}$ que comparou pacientes com angina estável tratados de forma invasiva ou conservadora, demonstrou que nos casos com tratamento clínico otimizado, sem alterações isquêmicas extensas nos exames funcionais, ambas as estratégias apresentaram resultados semelhantes, exceto pela qualidade de vida que foi melhor nos pacientes submetidos à ICP. Nesse estudo, $40 \%$ dos pacientes tinham mais de 65 anos (em média 72 anos) e concluiu-se que o tratamento clínico otimizado é efetivo nos pacientes maiores de 65 anos com angina estável e que a ICP não reduziu os eventos cardiovasculares ou ocorreu melhora da angina. ${ }^{38}$

\section{Cirurgia ou intervenção coronariana percutânea}

Dependendo da expectativa de vida, comorbidades e riscos de médio e longo prazo, a ICP vem se mostrando uma boa estratégia, mesmo para pacientes idosos com características anatômicas em que a sobrevida com a CRVM é significativamente maior. Atualmente, recomenda-se a CRVM como estratégia de revascularização para pacientes com doença multiarterial ou de tronco de coronária esquerda. Contudo, frente aos avanços da ICP com os stents farmacológicos, houve uma expansão das indicações da ICP, incluindo pacientes com lesões complexas, doença de tronco e multiarterial. Assim, a ICP é uma estratégia promissora em pacientes selecionados, principalmente nos idosos com alto risco para CRVM e múltiplas comorbidades. ${ }^{17}$

Uma metanálise comparou CRVM e ICP em pacientes multiarteriais e mostrou sobrevida semelhante, porém a sobrevida livre de IAM, AVE e revascularização de repetição em 5 anos favoreceu a CRVM (77\%) em relação à $\operatorname{ICP}(60 \%){ }^{39}$

Os octogenários com alto risco cirúrgico apresentam também um alto risco de eventos cardiovasculares associados à ICP. Isso deve ser considerado e devidamente esclarecido ao paciente quando é oferecida a ICP como alternativa à CRVM. ${ }^{25}$

Os médicos enfrentam um grande desafio ao definir a melhor estratégia de revascularização no idoso. Essa decisão deve ser embasada numa contextualização individual do paciente, apoiada em múltiplas variáveis e particularidades da população geriátrica.

\section{Referências}

1. Lourenço RA, Martins CSF, Sanchez MAS, Veras RP. Assistência ambulatorial geriátrica: hierarquização da demanda. Rev Saude Publica. 2005;39(2):311-8.

2. Lloyd-Sherlock P. Population ageing in developed and developing regions: implications for health policy. Soc Sci Med. 2000;51(6):887-95.

3. Laslett LJ, Alagona P, Jr., Clark BA 3rd, Drozda Jr JP, Saldivar F, Wilson SR, et al. The worldwide environment of cardiovascular disease: prevalence, diagnosis, therapy, and policy issues: a report from the American College of Cardiology. J Am Coll Cardiol 2012;60(25 Suppl):S1-49. http://dx.doi. org/10.1016/j.jacc.2012.11.002

4. Maranhão AGK. Como morrem os brasileiros: caracterização e distribuição geográfica dos óbitos no Brasil, 2000, 2005 e 2009. In: Brasil. Ministério da Saúde. Saúde Brasil 2010: Saúde Brasil 2010: uma análise da situação de saúde e de evidências selecionadas de impacto de ações de vigilância em saúde. Brasília: Ministério da Saúde; 2011. p. 51-78.

5. Aronow WS. Diagnosis and Management of Coronary Artery Disease. In: Tallis RC, Fillit HM. Brocklehurst's Textbook of Geriatric Medicine and Gerontology. 7th ed. Philadelphia: Sauders Elsevier; 2010. 
6. Peterson ED, Gharacholou SM. Coronary Heart Disease. In: Halter JB, Ouslander J, Tinetti M, Studenski S, High K, Asthana S Hazzard's Geriatric Medicine and Gerontology. 6th ed. New York: McGraw Hil Medical; 2009. p. 90920.

7. Vacanti LJ, Sposito AC, Sespedes L, Sarpi M, Ramires JA, Bortnick AE. In comparison to the myocardial perfusion scintigraphy, a treadmill stress test is a viable, efficient and cost effective option to predict cardiovascular events in elderly patients. Arq Bras Cardiol. 2007;88 (5):531-6.

8. Morrow DA, Gersh BJ, Libby P. Chronic coronary artery disease. Braunwald's heart disease: a textbook of cardiovascular medicine. 9th ed. Philadelphia: Elsevier Saunders; 2011. p. 1353-417.

9. Gravina CF, Franken $\mathrm{R}$, Wenger $\mathrm{N}$, Freitas EV, Batlouni M, Rich M, et al. [II Guidelines of Brazilian Society of Cardiology in geriatric cardiology]. Arq Bras Cardiol. 2010; 95 (3 Suppl 2):e16-76. II Diretrizes em Cardiogeriatria da Sociedade Brasileira de Cardiologia.

10. Siqueira BJ, Markman BF, Silva OB. Teste ergométrico em idosos. Geriatria \& Gerontologia 2011;5(1):40-5.

11. Ochiai ME, Gebara OC, Serro-Azul JB, Pinto LB, Nussbacher A, Pierri H, et al. [Exercise stress test: prognostic value for elderly patients with stable coronary atherosclerosis]. Arq Bras Cardiol 2006;86(5):346-52. Teste ergometrico: valor prognostico em pacientes idosos com doenca aterosclerotica coronariana estavel. http:// dx.doi.org/10.1590/S0066-782X2006000500004

12. Vacanti LJ, Sespedes LB, Sarpi MO. Exercise stress testing is useful, safe, and efficient even in patients aged 75 years or older. Arq Bras Cardiol. 2004;82(2):151-4.

13. Underwood SR, Anagnostopoulos C, Cerqueira M, Ell PJ, Flint EJ, Harbinson M, et al. Myocardial perfusion scintigraphy: the evidence. Eur J Nucl Med Mol Imaging. 2004;31(2):261-91. http:// dx.doi.org/10.1007/s00259-003-1344-5

14. Shaw LJ, Miller D. Noninvasive coronary risk stratification of elderly patients. Am J Geriatr Cardiol. 1994;3(1):12-21.

15. Steingart RM, Hodnett P, Musso J, Feuerman M. Exercise myocardial perfusion imaging in elderly patients. J Nucl Cardiol. 2002;9 (6):57380. http://dx.doi.org/10.1067/mnc.2002.127013

16. De Winter O, Van de Veire N, Gemmel F, Goethals I, De Sutter J. Myocardial perfusion imaging in the elderly: a review. Nucl Med Commun. 2006;27(6):529-34.

17. Liberman A, Wajngarten M. Doença coronariana crônica no idoso. In: Paola AAV, Barbosa
MM, Guimarães JI, editores. Livro Texto da Sociedade Brasileira de Cardiologia. São Paulo: Manole; 2012. p. 1565-72.

18. Tsang TS, Barnes ME, Gersh BJ, Takemoto Y, Rosales AG, Bailey KR, et al. Prediction of risk for first age-related cardiovascular events in an elderly population: the incremental value of echocardiography. J Am Coll Cardiol. 2003;42 (7):1199-205.

19. Douglas PS, Garcia MJ, Haines DE, Lai WW, Manning WJ, Patel AR, et al. ACCF/ ASE/AHA/ASNC/HFSA/HRS/SCAI/SCCM/ SCCT/SCMR 2011 Appropriate Use Criteria for Echocardiography A Report of the American College of Cardiology Foundation Appropriate Use Criteria Task Force, American Society of Echocardiography, American Heart Association, American Society of Nuclear Cardiology, Heart Failure Society of America, Heart Rhythm Society, Society for Cardiovascular Angiography and Interventions, Society of Critical Care Medicine, Society of Cardiovascular Computed Tomography, and Society for Cardiovascular Magnetic Resonance Endorsed by the American College of Chest Physicians. J Am Coll Cardiol. 2011;57 (9):112666. http://dx.doi.org/10.1016/j.jacc.2010.11.002

20. Anthopoulos LP, Bonou MS, Kardaras FG, Sioras EP, Kardara DN, Sideris AM, et al. Stress echocardiography in elderly patients with coronary artery disease: applicability, safety and prognostic value of dobutamine and adenosine echocardiography in elderly patients. J Am Coll Cardiol. 1996;28(1):52-9.

21. Pellikka PA. Stress echocardiography for the diagnosis of coronary artery disease: progress towards quantification. Current opinion in cardiology 2005;20(5):395-8.

22. Rochitte CE, Pinto IM, Fernandes JL, Filho CF, Jatene A, Carvalho AC, et al. [Cardiovascular magnetic resonance and computed tomography imaging guidelines of the Brazilian Society of Cardiology]. Arq Bras Cardiol. 2006; 87 (3): e60100. Diretrizes SBC: ressonância e tomografia cardiovascular.

23. Nandalur KR, Dwamena BA, Choudhri AF, Nandalur MR, Carlos RC. Diagnostic performance of stress cardiac magnetic resonance imaging in the detection of coronary artery disease: a meta-analysis. J Am Coll Cardiol. 2007;50(14):1343-53. http://dx.doi. org/10.1016/j.jacc.2007.06.030

24. Burgstahler C, Beck T, Kuettner A, Reimann A, Kopp AF, Heuschmid M, et al. Image quality and diagnostic accuracy of 16-slice multidetector spiral computed tomography for the detection of coronary artery disease in elderly patients. J Comput Assist Tomogr 2005;29(6):734-8. 
25. Sokol MC, McGuigan KA, Verbrugge RR, Epstein RS. Impact of medication adherence on hospitalization risk and healthcare cost. Med Care. 2005;43(6):521-30.

26. Yusuf S, Zhao F, Mehta SR, Chrolavicius S, Tognoni G, Fox KK. Effects of clopidogrel in addition to aspirin in patients with acute coronary syndromes without ST-segment elevation. N Engl J Med. 2001;345(7):494-502. http://dx.doi.org/10.1056/NEJMoa010746

27. Lucianno DP, Marliére AQ, Ribeiro MGL. Utilização de fitoterápicos por idosos: resultados de um inquérito domiciliar em Belo Horizonte (MG), Brasil. Rev Bras Farmacogn. 2008;18:754-60.

28. Freitas EV, Mohallen K, Gamarsk R. Recomendações da SOCERJ. Manejo Terapêutico em cardiogeriatria. Revista da SOCERJ. 2004;17(Supl B):1-96.

29. Yusuf S, Sleight P, Pogue J, Bosch J, Davies R, Dagenais G. Effects of an angiotensinconverting-enzyme inhibitor, ramipril, on cardiovascular events in high-risk patients. The Heart Outcomes Prevention Evaluation Study Investigators. N Engl J Med. 2000;342 (3):145-53. http://dx.doi.org/10.1056/ NEJM200001203420301

30. Heart Protection Study Collaborative Group. MRC/BHF Heart Protection Study of cholesterol lowering with simvastatin in 20,536 high-risk individuals: a randomised placebo-controlled trial. Lancet. 2002;360 (9326):7-22. http://dx.doi. org/10.1016/S0140-6736(02)09327-3

31. Shepherd J, Blauw GJ, Murphy MB, Bollen EL, Buckley BM, Cobbe SM, et al. Pravastatin in elderly individuals at risk of vascular disease (PROSPER): a randomised controlled trial. Lancet. 2002;360(9346):1623-30.

32. Schwartz GG, Olsson AG, Ezekowitz MD, Ganz P, Oliver MF, Waters D, et al. Effects of atorvastatin on early recurrent ischemic events in acute coronary syndromes: the MIRACL study: a randomized controlled trial. JAMA. 2001;285(13):1711-8.

33. Roach GW, Kanchuger M, Mangano CM, Newman M, Nussmeier N, Wolman R, et al. Adverse cerebral outcomes after coronary bypass surgery. Multicenter Study of Perioperative Ischemia Research Group and the Ischemia Research and Education Foundation Investigators. N Engl J Med. 1996;335(25):1857-63. http://dx.doi.org/10.1056/ NEJM199612193352501

34. Hannan EL, Wu C, Walford G, Culliford AT, Gold JP, Smith CR, et al. Drug-eluting stents vs. coronary-artery bypass grafting in multivessel coronary disease. N Engl J Med. 2008;358(4):331-41. http://dx.doi.org/10.1056/

\section{NEJMoa071804}

35. Ishikawa S, Buxton BF, Manson N, Hadj A, Seevanayagam S, Raman JS, et al. Cardiac surgery in octogenarians. ANZ J Surg. 2004;74 (11):983-5. http://dx.doi.org/10.1111/j.14451433.2004.03214.x

36. The TIME Investigators. Trial of invasive versus medical therapy in elderly patients with chronic symptomatic coronary-artery disease (TIME): a randomised trial. Lancet. 2001;358(9286):951-7. http://dx.doi.org/10.1016/S0140-6736(01)061001

37. Boden WE, O'Rourke RA, Teo KK, Hartigan PM, Maron DJ, Kostuk WJ, et al. Optimal medical therapy with or without PCI for stable coronary disease. N Engl J Med. 2007;356(15):1503-16. http://dx.doi.org/10.1056/NEJMoa070829

38. Teo KK, Sedlis SP, Boden WE, O'Rourke RA, Maron DJ, Hartigan PM, et al. Optimal medical therapy with or without percutaneous coronary intervention in older patients with stable coronary disease: a pre-specified subset analysis of the COURAGE (Clinical Outcomes Utilizing Revascularization and Aggressive druG Evaluation) trial. J Am Coll Cardiol. 2009;54 (14):1303-8. http://dx.doi.org/10.1016/j. jacc. 2009.07 .013

39. Daemen J, Boersma E, Flather M, Booth J, Stables R, Rodriguez A, et al. Long-term safety and efficacy of percutaneous coronary intervention with stenting and coronary artery bypass surgery for multivessel coronary artery disease: a meta-analysis with 5-year patient-level data from the ARTS, ERACIII, MASS-II, and SoS trials. Circulation. 2008;118(11):1146-54. http://dx.doi.org/10.1161/ CIRCULATIONAHA.107.752147 